\title{
Magnetic ordering effects in a Mn-modulation-doped high mobility two-dimensional hole system
}

\author{
Ursula Wurstbauer* \\ Institute of Applied Physics, University of Hamburg, D-20355 Hamburg, Germany \\ and Institute of Experimental and Applied Physics, University of Regensburg, D-93040 Regensburg, Germany \\ Werner Wegscheider ${ }^{\dagger}$ \\ Institute of Experimental and Applied Physics, University of Regensburg, D-93040 Regensburg, Germany
}

(Received 25 February 2009; published 28 April 2009)

\begin{abstract}
We have studied the magnetotransport properties of a manganese (Mn)-modulation-doped high mobility two-dimensional hole system in a strained InAs quantum well (QW) structure. At precisely $T=600 \mathrm{mK}$ a phase transition from paramagnetism to ferromagnetism can be observed by a change of the low-field magnetotransport behavior and hysteresis. In the magnetically ordered phase a superposition of positive magnetoresistance and weak antilocalization was detected in the longitudinal resistance $R_{x x}$ and in the Hall resistance $R_{x y}$ a superposition of normal, anomalous, and planar Hall effects demonstrating spontaneous magnetization in the QW plane was detected. From extensive analysis of the temperature and magnetic field dependence of the Shubnikov-de Haas oscillations we deduce the effective mass, transport, and quantum-scattering times. The latter indicates presence of small-range scattering potential. From corrections to the Drude conductivity we determine the impurity interaction time, which is significantly reduced in the ferromagnetic phase indicating interaction of the two-dimensional free holes' spin with the localized magnetic moments of 5/2 from Mn ions.
\end{abstract}

DOI: 10.1103/PhysRevB.79.155444

PACS number(s): 71.18.+y, 71.55.Eq, 72.15.Rn, 73.43.Qt

Magnetic semiconductor heterostructures derived from II-VI alloys with the transition metal Mn (Refs. 1-3) and diluted magnetic semiconductors such as GaMnAs, ${ }^{4}$ InMnAs ${ }^{5}$ and their quaternary compounds ${ }^{6}$ are currently studied intensely because of the interesting spin phenomena observed in these materials and their prospect for spinpolarized charge-carrier injection and manipulation in spintronic devices. The ferromagnetism in heavily $\mathrm{Mn}$ doped heterostructures is mediated by the $p$ - $d$ exchange interaction between the localized Mn spin of 5/2 and the spins of itinerant holes. ${ }^{7}$ By annealing GaMnAs or by integrating $\mathrm{Mn}$ into sophisticated GaAs-based heterostructures very high Curie temperatures higher than $170 \mathrm{~K}$ (Refs. 8 and 9) and $250 \mathrm{~K}$ (Ref. 10) have been reported. A lot of intriguing properties such as magnetic anisotropies ${ }^{11}$ and their device application potential ${ }^{12}$ as well as quantum interference effects ${ }^{13}$ have been explored based on these disordered magnetic conductors.

Recently, investigations on magnetic two-dimensional hole systems are reported ${ }^{14-16}$ in which the interplay of localized magnetic ions with the low-dimensional chargecarrier spins is studied. In these structures the $\mathrm{Mn}$ ions are in close proximity to the two-dimensional charge carriers. In contrast to magnetic two-dimensional electron systems, which have already been studied in II-VI materials, Mn plays a dual role in III-V heterostructures and provides both magnetic moments and free carriers. In both II-VI and III-V Mndoped two-dimensional charge-carrier systems, anomalous Hall effect, ${ }^{14,17}$ localization effects, ${ }^{1,16}$ and interesting behavior in the quantum-Hall regime ${ }^{2,3}$ have been observed.

It is known that ferromagnetic GaMnAs layers increase the spin lifetime of charge carriers in spatially separated nonmagnetic GaAs quantum wells (QWs). ${ }^{18}$ To investigate the interaction of magnetic moments in a low Mn doped layer with the spatially separated charge carriers of a high mobility two-dimensional hole system, we study Mn-modulation- doped QW structures without Mn ions in close proximity to the QW. We employ the small band-gap semiconductor InAs as the material hosting the magnetic two-dimensional hole gas (2DHG). InAs-based heterostructures offer several advantageous properties such as a large $g$ factor and large Rashba-induced spin-orbit coupling. ${ }^{19-21}$

For this experimental investigation high mobility $\mathrm{Mn}$ modulation-doped InAs $/ \operatorname{In}_{0.75} \mathrm{Ga}_{0.25} \mathrm{As} / \mathrm{In}_{0.75} \mathrm{Al}_{0.25} \mathrm{As}$ quantum well structures were grown by molecular-beam epitaxy on semi-insulating GaAs (001) substrates. The active layer is grown on a compositionally graded metamorphic buffer layer which leads to a virtual, on average, unstrained $\mathrm{In}_{0.75} \mathrm{Al}_{0.25} \mathrm{As}$ substrate. Details on the buffer layer growth are given in Refs. 22 and 23.

The active layer consists of a $20 \mathrm{~nm} \operatorname{In}_{0.75} \mathrm{Ga}_{0.25} \mathrm{As} \mathrm{QW}$ with an additional strained $4 \mathrm{~nm}$ InAs channel followed by a $5 \mathrm{~nm}$ thick $\operatorname{In}_{0.75} \mathrm{Al}_{0.25} \mathrm{As}$ spacer, a $7 \mathrm{~nm}$ thick $\mathrm{Mn}$ doped $\mathrm{In}_{0.75} \mathrm{Al}_{0.25} \mathrm{As}$ layer, and a $36 \mathrm{~nm} \operatorname{In}_{0.75} \mathrm{Al}_{0.25} \mathrm{As}$ cap layer. A schematic stack of the essential layers is shown in Fig. 1(c). From flux calibration of the Mn effusion cell a Mn acceptor concentration of less than $2 \times 10^{20} \mathrm{~cm}^{-3}$ is calculated. Transmission electron microscopy (TEM) reveals a good crystalline quality in the active region without indications of $\mathrm{Mn}$ cluster formation or MnAs inclusions. ${ }^{16}$ Further, secondaryion mass spectroscopy (SIMS) profiles exclude $\mathrm{Mn}$ in the QW or in the spacer due to back diffusion. ${ }^{16}$ The highly Mn-doped $\operatorname{In}_{0.75} \mathrm{Al}_{0.25} \mathrm{As}$ layer shows insulating behavior at low temperatures. Morphology investigations of the surface by atomic force microscopy reveal a characteristic crosshatched pattern of undulations aligned along the $\langle 110\rangle$ crystallographic directions as shown in Fig. 1(b). This is typical for lattice mismatched growth and is caused by the formation of a network of misfit dislocations aligned along the $\langle 110\rangle$ directions during the strain relaxation in the step graded buffer. $^{24}$ The root-mean-square (rms) roughness of our sample is 0.7 and $2.9 \mathrm{~nm}$ along the [110] and [110] direc- 


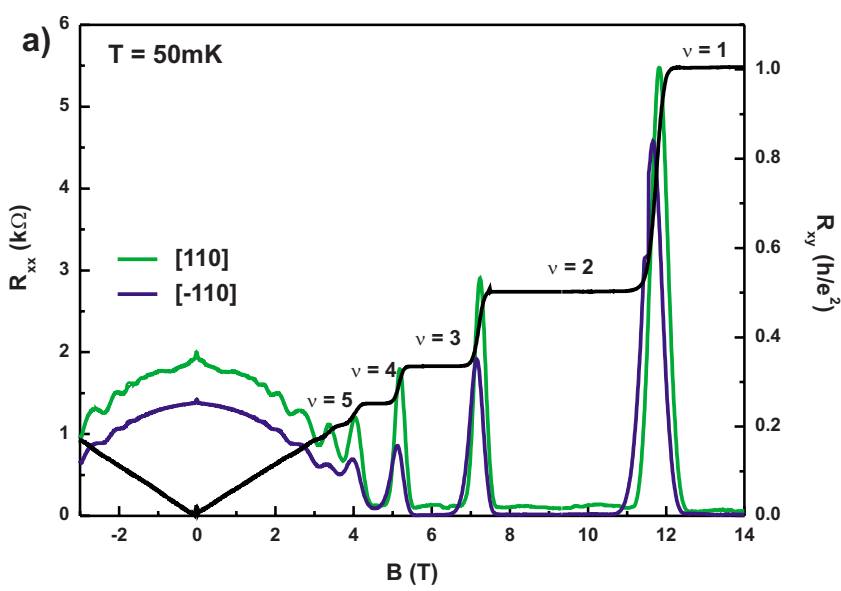

b)

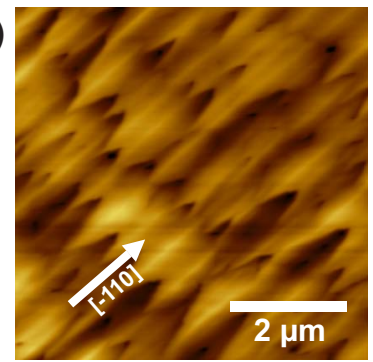

c)

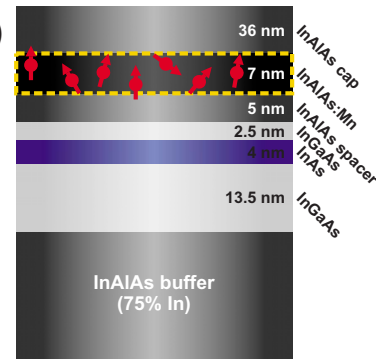

FIG. 1. (Color online) Hall resistance trace $R_{x y}$ (black) and longitudinal resistance $R_{x x}$ along the anisotropic [110] (blue) and [110] (green) directions at $T=50 \mathrm{mK}$ reveal clear quantum Hall plateaus and Shubnikov-de Haas oscillations with vanishing resistance indicating transport in two-dimensional system. (b) Atomic force microscopy (AFM) image of the cross-hatched morphology, which is anisotropic between the [110] and [110] directions. (c) Layer sequence of the active QW structure consisting of an InGaAs QW (light gray) with an asymmetrically embedded InAs channel (blue) and a Mn-doped InAlAs layer spaced from the QW by an InAlAs layer (dark gray.)

tions, respectively. This effect is mainly due to In concentration modulation, correlated with the surface morphology. ${ }^{24}$

For transport measurements L-shaped Hall bars have been fabricated along both the [110] and the [110] directions by optical lithography and wet chemical etching. The dimensions of the bars are $200 \mu \mathrm{m}$ for the width and $1000 \mu \mathrm{m}$ for the length. In/Zn alloyed at about $350{ }^{\circ} \mathrm{C}$ is used for preparation of electrical contacts with ohmic behavior. Lowtemperature magnetotransport measurements have been performed in a variable-temperature, pumped ${ }^{4} \mathrm{He}$ refrigerator and a ${ }^{3} \mathrm{He} /{ }^{4} \mathrm{He}$ dilution refrigerator allowing measurements in a wide temperature range from $30 \mathrm{mK}$ to $4.2 \mathrm{~K}$. The measurements were carried out using low-frequency lock-in technique with a bias current of $100 \mathrm{nA}$.

Figure 1(a) shows magnetotransport measurements of the Hall resistance $R_{x y}$ (black trace) and the longitudinal resistance $R_{x x}$ along the [110] (blue) and [110] (green) directions at $T=30 \mathrm{mK}$. A marked difference in the mobility and the magnetoresistance between the [110] and [1 10] directions is observed corresponding to the differences of the surface morphology. The onset of Shubnikov-de Haas $(\mathrm{SdH})$ oscillations at about $B=1 \mathrm{~T}$ in $R_{x x}$ and well-pronounced Hall plateaus in $R_{x y}$ validate charge-carrier transport in a high mobility two-dimensional system. The positive Hall coefficient confirms transport in a two-dimensional hole system. The vanishing resistances at filling factors $\nu=3, \nu=2$, and $\nu=1$ for both directions exclude the presence of a parallel conduction channel. Hall plateaus are visible at odd and even filling factors up to filling factor $\nu=7$ in the Hall trace. The two-dimensional (2D)-hole density was determined to be $p$ $=4.4 \times 10^{11} \mathrm{~cm}^{-2}$ from low-field Hall measurements and confirmed by the $1 / B$ periodicity of the $\mathrm{SdH}$ oscillations in the whole temperature range. The mobility at $T=4.2 \mathrm{~K}$ is $11.2 \times 10^{3}$ and $8.3 \times 10^{3} \mathrm{~cm}^{2} / \mathrm{V} \mathrm{s}$ for the [110] and the [110] directions, respectively. The detected anisotropy in the mobility constitutes $23 \%$ between the orthogonal $\langle 110\rangle$ directions. The anisotropy is well known from twodimensional electron gases in such metamorphic InAs QW structures. ${ }^{24,25}$

Figures 2(a) and 2(b) show the longitudinal magnetoresistance $R_{x x}(B)$ along the [110] and the [110] directions for different temperatures in the milli-Kelvin region. In the field range from -3 to $3 \mathrm{~T}$ the $\mathrm{SdH}$ oscillations are superimposed by a negative parabolic magnetoresistance background. This can be attributed for two-dimensional charge-carrier systems to quantum corrections to the classical Drude conductivity. ${ }^{26}$ In addition, for two-dimensional hole systems, it may arise also from the presence of intersubband scattering. ${ }^{27,28}$ The latter seems not to be the reason for the parabolic magnetoresistance. From the measurements we see no indications for contributions of two different subbands such as beating patterns or two $1 / B$ frequencies from fast-Fouriertransformation (FFT) analysis of the reciprocal $\mathrm{SdH}$ oscillations. ${ }^{29}$ For high mobility two-dimensional chargecarrier systems, e.g., in III-V heterostructures, there exists a range of $B$ (at higher fields) that shows preferentially the effect of electron-electron interaction (EEI) in the diffusion channel. ${ }^{26}$ The effect caused by EEI has also been observed on high mobility 2DEGs in GaAs/AlGaAs (Refs. 26 and 30) and AlGaN/GaN (Ref. 31) heterostructures and one can take advantage of the temperature dependence of this effect to determine the electron-electron interaction time $\tau_{\mathrm{ee}}$ as discussed later.

In order to study the low-field region of the magnetoresistance and the $T$ - and $B$-dependent dampings of the $\mathrm{SdH}$ oscillations, the parabolic background was subtracted from the longitudinal magnetoresistance $R_{x x}(B)$ in the field range of $\pm 3 \mathrm{~T}$ as depicted in Figs. 2(c) and 2(d) for the [ $\overline{1} 10]$ and the [110] directions. For the determination of the effective mass and the quantum-scattering times in 2D charge-carrier systems with only one occupied subband, the variation in the amplitude $\Delta R_{x x}(B)$ of the $\mathrm{SdH}$ oscillation with temperature and magnetic field is used. The effective mass $m^{*}$ was detected by fitting $\Delta R_{x x}(B)$ to the factor $\chi / \sinh (\chi)$, with $\chi$ $=2 \pi k_{B} T /\left(\hbar \omega_{c}\right)$ and $\omega_{c}=e B / m^{*} \cdot 32,33$ We followed Elhamri and co-workers and approximated $\sinh (\chi)$ with $\exp (\chi) / 2 .{ }^{34}$ The amplitude $\Delta R_{x x}(B)$ of the $\mathrm{SdH}$ oscillation at a given magnetic field can be expressed as 

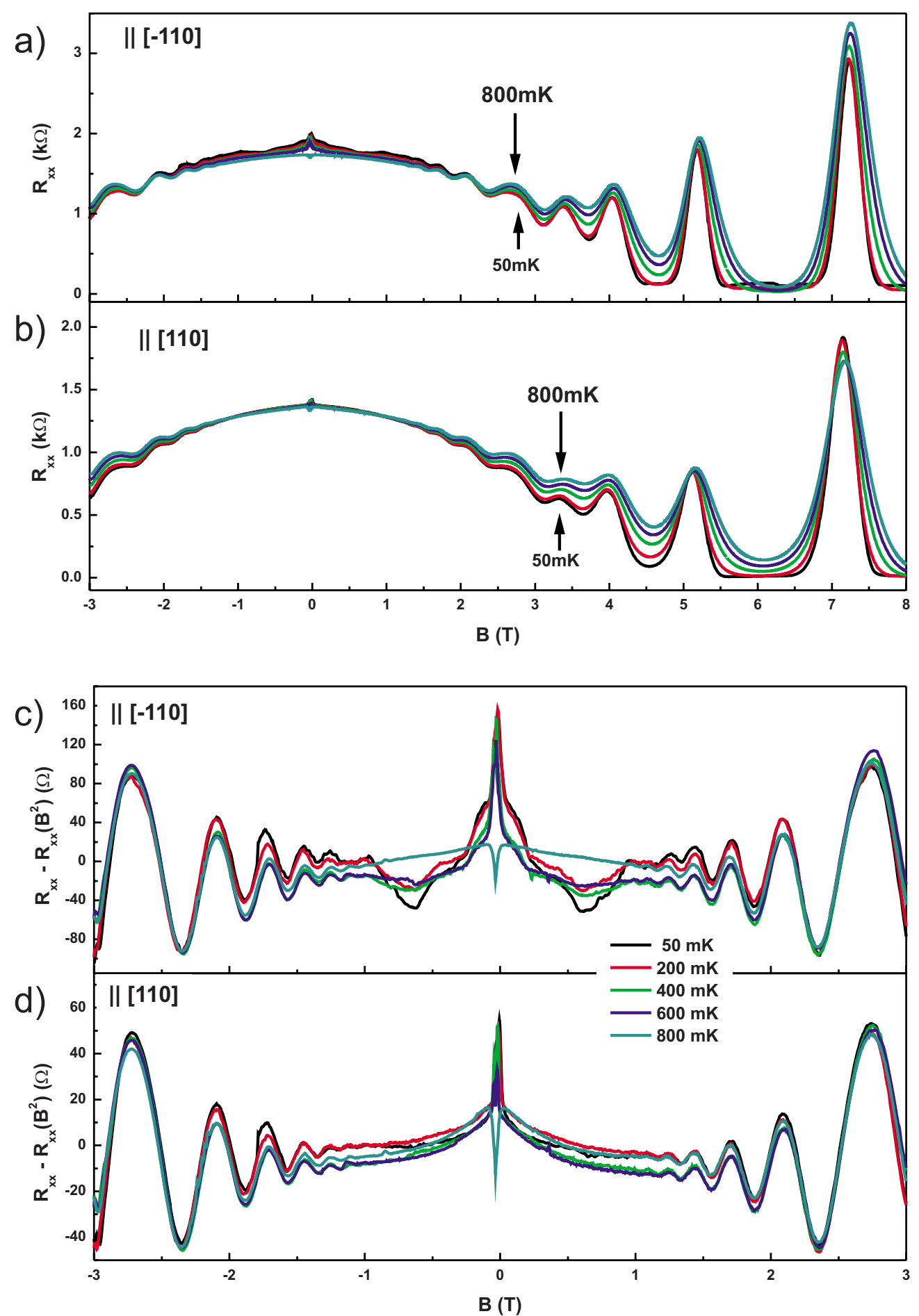

FIG. 2. (Color online) Longitudinal magnetoresistance traces $R_{x x}(B)$ in the low-field region for temperatures between 50 and $800 \mathrm{mK}$ along the (a) [ $\overline{1} 10]$ and the (b) [110] directions. [(c) and (d)] Resistance traces from (a) and (b) obtained after subtraction of the second-order polynomial background demonstrating the strong $T$ dependence of the magnetoresistance around $B=0$.

$$
\ln \left[\Delta R_{x x}(B) / T\right] \approx C-\frac{2 \pi^{2} k_{B} m^{*}}{e \hbar B} T
$$

where $C$ is a temperature-independent term. A plot of $\ln \left[\Delta R_{x x}(B) / T\right]$ versus $T$ gives a straight line with a slope of $\left(\frac{2 \pi^{2} k_{B} m^{*}}{e \hbar B}\right)$ from which the effective mass $m^{*}(B)$ for fixed $B$ values can be calculated. We determined $m^{*}(B)$ from temperature-dependent measurements of the $\mathrm{SdH}$ oscillations between 30 and $800 \mathrm{mK}$ in the field range from 2 to $4 \mathrm{~T}$. When we plot the values for $m^{*}(B)$ versus $B$ which give straight lines for the two orthogonal $\langle 110\rangle$ directions, linear 
extrapolation of our data to $B=0$ suggests $m_{[-110]}^{*} \approx 0.13 m_{0}$ and $m_{[110]}^{*} \approx 0.17 m_{0}$, respectively. The slope of the linear extrapolation is almost identical along the two directions and differs slightly from zero. The anisotropy of the effective mass is for the (001) plane unexpected and can be associated with the differences of the modulation in the In concentrations leading to an anisotropic modulation in the potential landscape. Due to the lattice mismatch between $\mathrm{In}_{0.75} \mathrm{Ga}_{0.25} \mathrm{As}$ and InAs, the InAs QW is compressively strained. The strain involves a splitting between heavy hole (hh) and light (lh) hole bands at the Brillouin-zone center $^{35}$ resulting in a mixing of hh and $\mathrm{lh}$ states with increasing $\vec{k} .^{36}$ The theoretically calculated value for the effective hole masses in InAs is $m_{\mathrm{lh}}^{*}=0.025 m_{0}$ for the $\mathrm{lh}$ band and $m_{\mathrm{hh}}^{*}=0.41 m_{0}$ for the hh band at the $\Gamma$ point. ${ }^{37}$ The deviation of experimentally observed values can be explained by nonparabolicity and mixing of the hh and lh valence bands. $^{38}$

The quantum-scattering time $\tau_{q}$ is given by the total scattering rate contrary to the classical scattering $\tau_{t}$ time which is weighted by the scattering angle $\Theta .{ }^{39}$ These two times are identical for short-range potentials, whereas the latter is enhanced in long-range potentials corresponding to small-angle scattering events. ${ }^{39}$ The classical scattering time $\tau_{t}$ can be determined with the above values of $m^{*}$ from the mobility $\mu=e \tau_{t} / m^{*}$ to about $0.79 \mathrm{ps}$ for the $[\overline{1} 10]$ direction and to about 0.8 ps for the [110] direction. The quantum-scattering time $\tau_{q}$ can be obtained from the amplitude $\Delta R_{x x}(B)$ of the $\mathrm{SdH}$ oscillation at a given temperature by evaluating the slope of the straight line $33,34,39$

$$
\ln \left(\frac{1}{4} \frac{\Delta R_{x x}}{R_{0}} \frac{\sinh \chi}{\chi}\right)=C-\left(\frac{\pi m^{*}}{e \tau_{q}}\right) \frac{1}{B}
$$

We determine the quantum-scattering time again for temperatures between 30 and $800 \mathrm{mK}$. For the [110] direction we obtain $\tau_{q,[110]} \approx 0.30 \mathrm{ps}$ and for the [110] direction $\tau_{q,[110]} \approx 0.38 \mathrm{ps}$. No significant temperature dependence of $\tau_{q}$ was found. The $\tau_{t} / \tau_{q}$ ratio is about 2.7 for the [110] direction and about 2.1 for the [110] direction, indicating a relatively high percent of large-angle scattering.

As mentioned above, the impurity interaction time, here the hole-hole interaction time $\tau_{\text {hh }}$ can be determined from the superimposed parabolic background to the longitudinal magnetoresistance $R_{x x}(B)$ in the intermediate field range up to $|B|=3$ T. To evaluate the values for $\tau_{\text {hh }}$ we follow the procedure introduced by Choi and co-workers. ${ }^{26}$ The parabolic background can be written as

$$
\Delta \rho_{x x}(B) \propto \rho B^{2} \delta \sigma_{\mathrm{hh}} .
$$

From perturbation theory, there is a correction $\left(\delta \sigma_{\mathrm{hh}}\right)$ to the Drude conductivity $\left(\sigma_{0}\right)$ due to electron-electron (here holehole) interaction in the diffusion channel in the metallic regime $\left(E_{F} \tau_{\mathrm{hh}} / \hbar \gg 1\right)$. For $k_{B} T \tau_{\mathrm{hh}} / \hbar<1$ the theory predicts in 2D (Ref. 26)
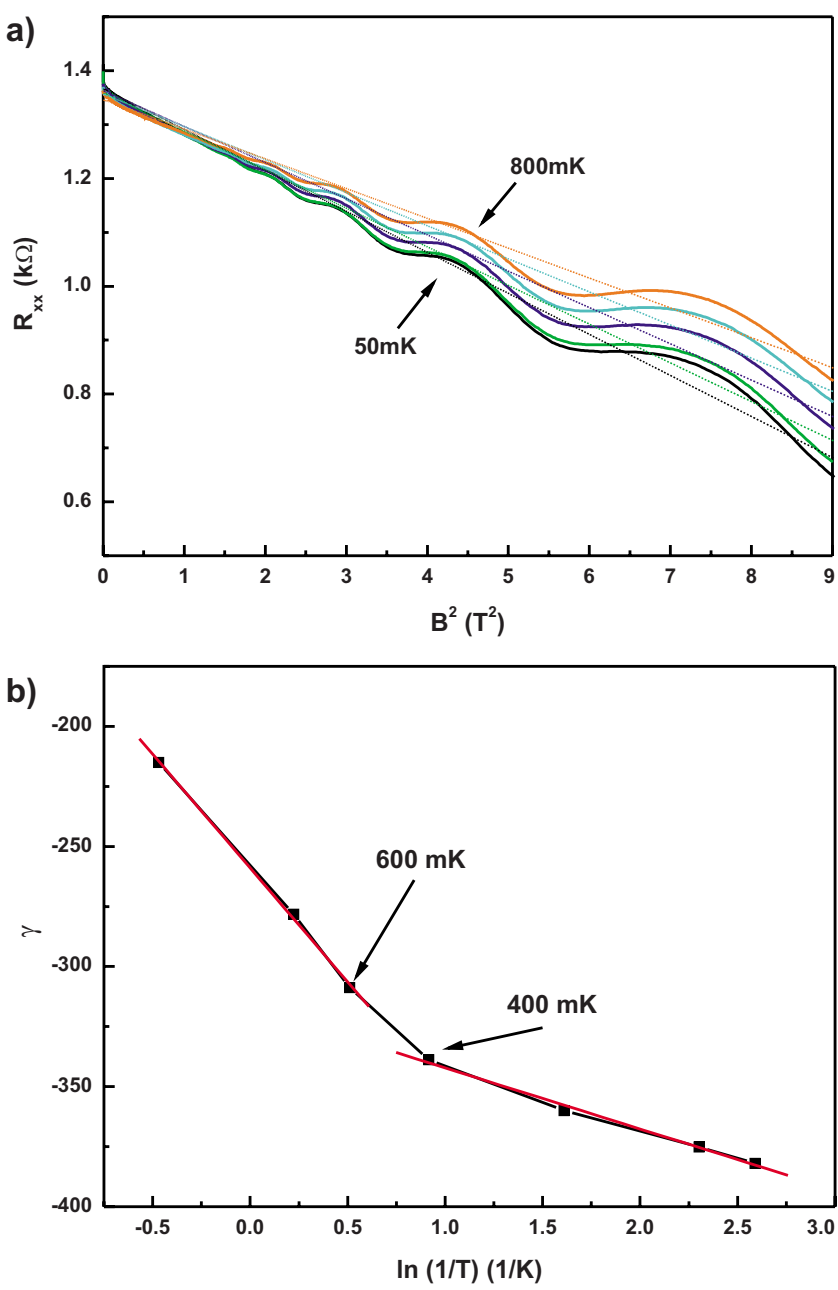

FIG. 3. (Color online) (a) Magnetoresistance $R_{x x}(B)$ (solids) and second-order polynomial fit (dashes) as a function of $B^{2}$ along the [110] direction for five temperatures: 50, 200, 400, 600, and 800 $\mathrm{mK}$. (b) Linear slope $\gamma$ from (a) plotted vs $\ln (1 / T$ ) (from $50 \mathrm{mK}$ to $1.5 \mathrm{~K})$. The slope gives the impurity scattering time $\tau_{\mathrm{hh}}$, which changes at $T=600 \mathrm{mK}$.

$$
\delta \sigma_{\mathrm{hh}}=-\left[4-3 \frac{2+F}{F} \ln \left(1+\frac{F}{2}\right)\right]\left(\frac{e^{2}}{2 \pi^{2} \hbar}\right) \ln \left(\frac{\hbar}{k_{B} T \tau_{\mathrm{hh}}}\right),
$$

where $F=\int \frac{d \Theta}{2 \pi}\left[1+\frac{2 k_{F}}{\kappa} \sin \left(\frac{\theta}{2}\right)\right]^{-1}, k_{F}$ is the Fermi wave vector, and $\kappa$ is the inverse screening length in $2 \mathrm{D} .{ }^{26}$ To ascertain the EEI in our structure, $R_{x x}(B)$ is plotted as a function of $B^{2}$ for different temperatures from $50 \mathrm{mK}$ to $1.5 \mathrm{~K}$ as demonstrated in Fig. 3(a) for the [110] direction. The parabolic background is fitted with a second-order polynomial (dashed lines). The slope $\gamma$ is a function of the temperature and is plotted as a function of $\ln (1 / T)$ as depicted in Fig. 3(b) for the [110] direction. From this dependence the impurity interaction time $\tau_{\mathrm{hh}}$ can be calculated using Eqs. (3) and (4). Surprisingly, from this plot we get two different slopes: one for temperatures from 50 to $400 \mathrm{mK}$ and one for temperatures from $600 \mathrm{mK}$ to $1.5 \mathrm{~K}$ indicating different interaction mechanisms in the two ranges. An identical behavior is ob- 
TABLE I. Numerical parameters obtained from analysis of the low-temperature low-field transport coefficients: effective mass $m^{*}$, transport $\tau_{t}$, quantum lifetime $\tau_{q}$, ratio $\tau_{t} / \tau_{q}$, and impurity scattering times $\tau_{\mathrm{hh},>}$ for $T \geq 600 \mathrm{mK}$ and $\tau_{\mathrm{hh},<}$ for $T<600 \mathrm{mK}$.

\begin{tabular}{cccccccc}
\hline \hline Direction & $\begin{array}{c}\mu \\
\left(\mathrm{cm}^{2} / \mathrm{Vs}\right)\end{array}$ & $\begin{array}{c}m^{*} \\
\left(m_{0}\right)\end{array}$ & $\begin{array}{c}\tau_{t} \\
(\mathrm{ps})\end{array}$ & $\begin{array}{c}\tau_{q} \\
(\mathrm{ps})\end{array}$ & $\tau_{t} / \tau_{q}$ & $\begin{array}{c}\tau_{\mathrm{hh},>>} \\
(\mathrm{fs})\end{array}$ & $\begin{array}{c}\tau_{\mathrm{hh},<} \\
(\mathrm{fs})\end{array}$ \\
\hline$[\overline{1} 10]$ & 11200 & 0.13 & 0.79 & 0.3 & 2.7 & 6.6 & 2.9 \\
{$[110]$} & 8300 & 0.17 & 0.8 & 0.38 & 2.1 & 5.5 & 2.85 \\
\hline \hline
\end{tabular}

served for the [1110] direction (not shown). The corresponding interaction times are for temperatures up to $T=400 \mathrm{mK}$ $\tau_{\mathrm{hh},<} \approx 2.9$ fs for the $[\overline{1} 10]$ and $\tau_{\mathrm{hh},<} \approx 2.85$ fs for the [110] direction and for temperatures from $600 \mathrm{mK}$ to $1.5 \mathrm{~K} \tau_{\mathrm{hh},>}$ $\approx 6.6 \mathrm{fs}$ and $\tau_{\mathrm{hh},>} \approx 5.5 \mathrm{fs}$ for the $[\overline{1} 10]$ and $[110]$ directions, respectively. The values for $\tau_{\mathrm{hh},<}$ in the lowtemperature range are isotropic, whereas at higher temperatures $\tau_{\mathrm{hh},>}$ show an $20 \%$ anisotropy between the orthogonal $\langle 110\rangle$ crystallographic directions. The asymmetry is comparable to the anisotropy of the mobility $\mu$, the quantumscattering time $\tau_{q}$, effective mass $m^{*}$, and the surface roughness. The values for mobility, effective mass, and the scattering and interaction times are summarized in Table I. For InGaAs/InAs alloys the most dominant scattering mechanisms are isotropic scattering on background impurities, remote impurities, and anisotropic scattering on alloy disorder, interface roughness, and on valence-band energy modulations due to In compositional fluctuations. ${ }^{24,40} \mathrm{We}$ associate the anisotropy in mobility, effective mass, quantumscattering time $\tau_{q}$, and the impurity interaction time for $T$ $\geq 600 \mathrm{mK} \tau_{\mathrm{hh},>}$ with the valence-band energy modulation correlated with the In concentration fluctuations. ${ }^{24}$ The isotropic transport scattering time that is dependent on smallangle scattering events seems to be dominated by the isotropic remote impurity scattering on the spaced $\mathrm{Mn}$ ions in the InAlAs Mn doped layer.

In order to understand the behavior of the impurity scattering time $\tau_{\mathrm{hh}}$, we investigate the low-field region of the longitudinal magnetoresistance. The resistance measurements after subtraction of the parabolic background shown in Figs. 2(c) and 2(d) point out a strong asymmetry between the $[\overline{1} 10]$ and the $[110]$ crystal directions and a strong temperature dependence for fields below $|B|<1 \mathrm{~T}$.

The strongest changes in $R_{x x}$ appear in the vicinity of $B$ $=0$. Figure $4(\mathrm{a})$ shows the magnetoresistance exemplarily for the [110] crystallographic direction at temperatures from 50 $\mathrm{mK}$ to $1.5 \mathrm{~K}$ for fields below $|B|<300 \mathrm{mT}$. The magnetic field was always swept from negative to positive field values. A clear transition from the trace corresponding to $T$ $=600 \mathrm{mK}$ to the trace corresponding to $T=610 \mathrm{mK}$ is observed. At $T=610 \mathrm{mK}$ a positive signal up to about $40 \mathrm{mT}$ appears followed by a negative magnetoresistance. The minimum of the resistance trace is located exactly at $B=0 \mathrm{~T}$. We interpret the magnetoresistance at $T=610 \mathrm{mK}$ and above as weak antilocalization (WAL) effects. This assumption is confirmed by the exact position of the minimum at $B=0 \mathrm{~T}$ and the decrease of the effect with increasing temperature. The WAL minimum vanishes above $T=4.2 \mathrm{~K}$ (not shown). WAL effects have also be seen in high mobility 2DEGS in comparable InAs QW structures ${ }^{21}$ and refer to a very strong spinorbit coupling of the charge-carrier systems in these QW structure.

In contrast, the resistance traces for $T=600 \mathrm{mK}$ and below clearly reveal a distinct rise in the magnetoresistance traces at low fields with an additional minimum close to $B$ $=0 \mathrm{~T}$ as demonstrated in Fig. 4(a). The effect increases with
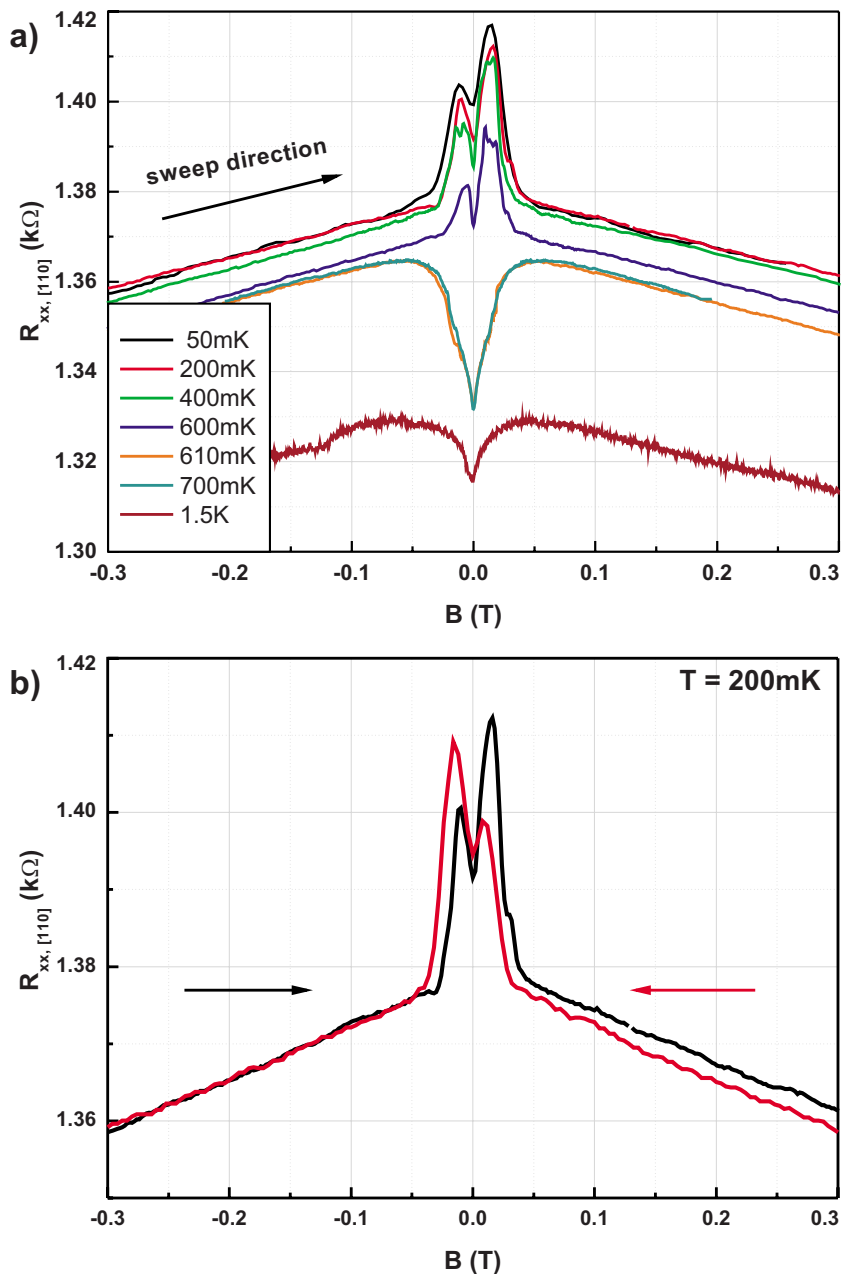

FIG. 4. (Color online) (a) Magnetoresistance in the vicinity of $B=0$ for the $[110]$ direction for temperatures ranging from $T$ $=50 \mathrm{mK}$ to $T=800 \mathrm{mK}$. The sign of the magnetoresistance changes between $T=610 \mathrm{mK}$ (blue) and $T=600 \mathrm{mK}$ (orange) from a weak antilocalizationlike behavior to a hystereticlike rise in the magnetoresistance with a minimum at $B=0 \mathrm{~T}$. (b) Up (black) and down (red) sweeps of the $T=200 \mathrm{mK}$ trace from (a) indicate hysteretic behavior. 
decreasing temperature. As shown for $T=200 \mathrm{mK}$ in Fig. 4(b), a clear hysteresis between up (black curve) and down (red curve) sweeps is observable. The additional minimum at $B=0 \mathrm{~T}$ exhibits no hysteretic behavior and can be interpreted as a superposition of the hysteretic rise and WAL effect. The sharp change in the transport-behavior within only $10 \mathrm{mK}$ and the hysteretic behavior for $T \leq 600 \mathrm{mK}$ point toward a phase transition from paramagnetism to a magnetically ordered phase. To confirm this assumption we investigate the temperature dependence of the Hall resistance traces $R_{x y}$ in the low-field region as plotted in Fig. 5(a). Around $B=0 \quad \mathrm{~T}$ the Hall resistance traces show a maximum for temperatures below $T \leq 600 \mathrm{mK}$. A transition in the behavior from $T=600 \mathrm{mK}$ (blue) to the trace at $T=610 \mathrm{mK}$ (orange) appears in correspondence with the behavior of the longitudinal resistance $R_{x x}$. For higher temperatures a straight line is observed with some noise in $R_{x y}$ in the vicinity of zero field. Slight changes in the slopes appear with increasing temperatures. This can be explained as a superposition of anomalous and normal Hall effects which can contribute to $R_{x y}$ also in nonferromagnetic $2 \mathrm{D}$ systems. ${ }^{17}$ The contribution of the anomalous Hall effect to $R_{x y}$ is temperature dependent. For $T \geq 1.5 \mathrm{~K}$ the slope of $R_{x y}$ in the lowfield region is in good agreement with the $2 \mathrm{D}$ hole density $p$ determined from the $\mathrm{SdH}$ measurements indicating vanishing contribution of the anomalous Hall effect. In Fig. 5(b) up (black) and down (red) sweeps of the Hall resistance $R_{x y}$ for $T=200 \mathrm{mK}$ indicating hysteretic behavior are shown. After subtraction of a straight line with the slope of normal Hall resistance corresponding to the $\mathrm{SdH}$ hole density of $p=4.4$ $\times 10^{11} \mathrm{~cm}^{-2}$, the traces reveal the typical shape of the planar Hall effect (PHE). ${ }^{41}$ This is demonstrated in Fig. 5(c) for $T$ $=200 \mathrm{mK}$ for both sweep directions. The measured $R_{x y}$ below $T \leq 600 \mathrm{mK}$ is a superposition of normal, anomalous, and planar Hall effects.

From the perfectly reproducible transition temperature at $T=600 \mathrm{mK}$ and the hysteretic behavior below this temperature in $R_{x x}$ and $R_{x y}$ we infer a phase transition from the paramagnetic to a ferromagnetic phase. Due to the fact that magnetic impurities are only in the Mn doped InAlAs layer, 7.5 $\mathrm{nm}$ spaced from the InAs QW, and a contamination of the InAs QW with Mn is excluded from SIMS measurements, ${ }^{16}$ we expect the InAlAs:Mn to become magnetically ordered below the Curie temperature $T_{C}=600 \mathrm{mK}$. The PHE indicates a spontaneous magnetization in the (001) plane. Hence, the minimum in the positive magnetoresistance in $R_{x x}$ in the ferromagnetic phase can be attributed to the identical phenomenon as in the paramagnetic phase, where this behavior is attributed to WAL effects. We interpret the double maxima [see Fig. 4(a)] to a superposition of anisotropic magnetoresistance due to the in-plane magnetization and WAL effect due to the overlaying perpendicular applied $B$ field. The change of value and symmetry properties in the impurity interaction times $\tau_{\text {hh }}$ at $T=600 \mathrm{mK}$ and $T=400 \mathrm{mK}$ can be explained as a penetration of the wave function of the free two-dimensional holes into the Mn-doped layer in the barrier. The change from anisotropic to isotropic behavior implies a change of the dominant interaction process. The reduced values of $\tau_{\mathrm{hh}}$ at low temperatures indicate a stronger interaction between the holes which should be spin polarized

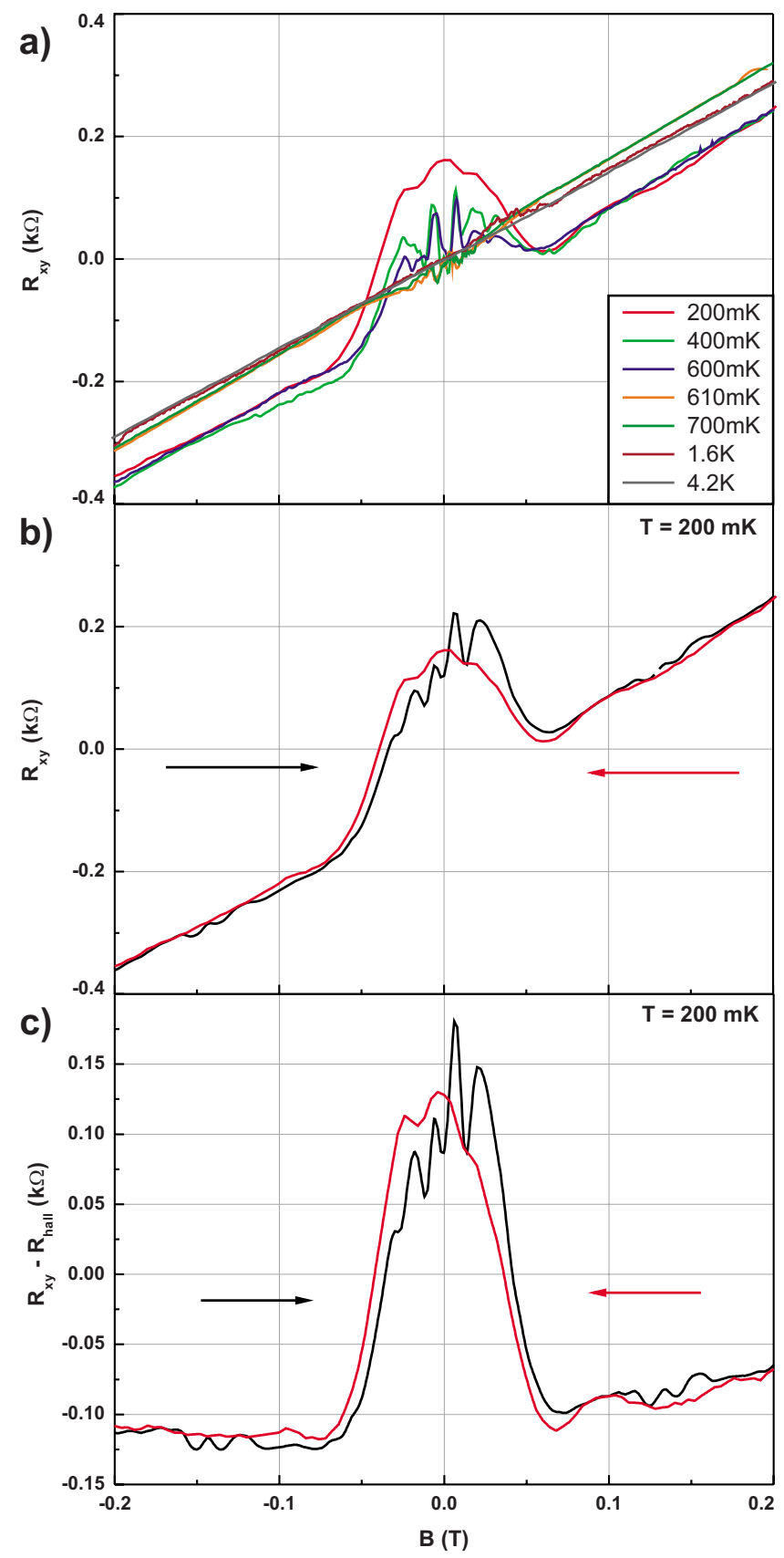

FIG. 5. (Color online) (a) Hall resistance traces $R_{x y}$ in the lowfield region for temperatures ranging from $200 \mathrm{mK}$ to $4.2 \mathrm{~K}$. The traces for $T \leq 600 \mathrm{mK}$ show an additional feature around $B=0$ and are shifted vertically compared to the traces for $T>600 \mathrm{mK}$. (b) Up (black) and down (red) sweeps of the Hall measurements at $T$ $=200 \mathrm{mK}$ show hysteretic behavior. (c) Up and down sweeps at $T=200 \mathrm{mK}$ depict typical trace of the planar Hall effect after subtraction of the normal Hall resistance trace corresponding to the SdH hole density.

due to the overlap of the wave function to the magnetically ordered InAlAs:Mn layer in the ferromagnetic phase. The role of the free $2 \mathrm{D}$ holes for the appearance of the holemediated ferromagnetism is still unclear. The 2D holes either participate direct to the exchange coupling between the Mn spins of $5 / 2$ or the magnetic ordering effect is mediated by bound holes in the InAlAs:Mn layer and the itinerant 2D 
holes interact with these 3D holes. In this context we want to point out that we see no parallel conductance from the transport experiments. Therefrom, we exclude at these low temperatures the presence of free holes in the InAlAs:Mn layer.

In conclusion, we have investigated scattering times, effective masses, and ordering effects in a high mobility Mnmodulation-doped two-dimensional hole system in a strained InAs QW. The high quality of the $2 \mathrm{DHG}$ can be seen from well-pronounced Hall plateaus, onset of the $\mathrm{SdH}$ oscillations at $B \approx 1 \mathrm{~T}$, and the vanishing $R_{x x}$ for filling factor $\nu=1,2$, and 3 . The anisotropy in nearly all transport coefficients and effective masses between the orthogonal $\langle 110\rangle$ directions is mainly caused by valence-band energy modulations due to In compositional fluctuations. ${ }^{24}$ The effective masses $m_{[\overline{110]}}^{*}=0.13 m_{0}$ for the [110] direction and $m_{[110]}^{*}=0.17 m_{0}$ for the [110] direction indicate valence-band nonparabolicity arising from interaction of hh and $\mathrm{lh}$ bands. The ratio $\tau_{t} / \tau_{q}$ is close to unity for both directions characteristic for a shortrange scattering potential. From the parabolic background around $|B|<3 \mathrm{~T}$ in $R_{x x}$ the impurity interaction time $\tau_{\mathrm{hh}}$ was determined, which is anisotropic above $T=600 \mathrm{mK}$ and reduced and isotropic below. Below the transition temperature at $T_{c}=600 \mathrm{mK}$ the system is ferromagnetic indicated by hysteresis and a clear change in the low-field transport behavior in both $R_{x x}$ and $R_{x y}$. The measured Hall resistance $R_{x y}$ in this temperature range is a superposition of normal, anomalous, and planar Hall effects. The latter demonstrates that the spontaneous magnetization direction lies in the (001) plane. In the longitudinal resistance anisotropic positive magnetoresistance was observed with a superimposed minimum. The minimum appears in the paramagnetic as well as in the ferromagnetic phase exactly at $B=0 \mathrm{~T}$. From the temperature dependence this effect is interpreted to be weak antilocalization.

We thank W. Hansen for stimulating discussions and D. Schuh for technical support. This work was supported by the DFG via Grant Nos. SFB 508 and 689. *ursula.wurstbauer@physik.uni-hamburg.de

†Present address: Solid State Physics Laboratory, ETH Zurich, 8093 Zurich, Switzerland.

${ }^{1}$ R. Knobel, N. Samarth, J. G. E. Harris, and D. D. Awschalom, Phys. Rev. B 65, 235327 (2002).

${ }^{2}$ J. Jaroszynski, T. Andrearczyk, G. Karczewski, J. Wrobel, T. Wojtowicz, D. Popovic, and T. Dietl, Phys. Rev. B 76, 045322 (2007).

${ }^{3}$ I. P. Smorchkova, N. Samarth, J. M. Kikkawa, and D. D. Awschalom, Phys. Rev. B 58, R4238 (1998).

${ }^{4} \mathrm{H}$. Ohno, Science 14, 281 (1998).

${ }^{5}$ T. Schallenberg and H. Munekata, Appl. Phys. Lett. 89, 042507 (2006).

${ }^{6}$ S. Ohya, H. Kobayashi, and M. Tanaka, Appl. Phys. Lett. 83, 2175 (2003).

${ }^{7}$ J. Fabian, A. Matos-Abiague, C. Ertler, and P. Stano, Acta Phys. Slov. 57, 565 (2007)

${ }^{8}$ K. Olejnik, M. H. S. Owen, V. Novak, J. Masek, A. C. Irvine, J. Wunderlich, and T. Jungwirth, Phys. Rev. B 78, 054403 (2008).

${ }^{9}$ K. W. Edmonds, P. Boguslawski, K. Y. Wang, R. P. Campion, S. N. Novikov, N. R. S. Farley, B. L. Gallagher, C. T. Foxon, M. Sawicki, T. Dietl, M. B. Nardelli et al., Phys. Rev. Lett. 92, 037201 (2004).

${ }^{10}$ A. M. Nazmul, T. Amemiya, Y. Shuto, S. Sugahara, and M. Tanaka, Phys. Rev. Lett. 95, 017201 (2005).

${ }^{11}$ C. Bihler, M. Althammer, A. Brandlmaier, S. Geprägs, M. Weiler, M. Opel, W. Schoch, W. Limmer, R. Gross, M. S. Brandt et al., Phys. Rev. B 78, 045203 (2008).

${ }^{12}$ K. Pappert, S. Hümpfner, C. Gould, J. Wenisch, K. Brunner, G. Schmidt, and L. W. Molenkamp, Nat. Phys. 3, 573 (2007).

${ }^{13}$ D. Neumaier, K. Wagner, U. Wurstbauer, M. Reinwald, W. Wegscheider, and D. Weiss, New J. Phys. 10, 055016 (2008).

${ }^{14}$ B. A. Aronzon, M. V. Kovalchuk, E. M. Pashaev, M. A. Chuev, V. V. Kvardakov, I. A. Subbotin, V. V. Rylkov, M. A. Pankov, I. A. Likhachev, B. N. Zvonkov et al., J. Phys.: Condens. Matter 20, 145207 (2008).
${ }^{15}$ U. Wurstbauer, I. Gronwald, U. Stöberl, A. Vogl, D. Schuh, D. Weiss, and W. Wegscheider, Physica E (Amsterdam) 40, 1563 (2008).

${ }^{16}$ U. Wurstbauer, M. Soda, R. Jakiela, D. Schuh, D. Weiss, and W. Wegscheider, J. Cryst. Growth 311, 2160 (2009).

${ }^{17}$ J. Cumings, L. S. Moore, H. T. Chou, K. C. Ku, G. Xiang, S. A. Crooker, N. Samarth, and D. Goldhaber-Gordon, Phys. Rev. Lett. 96, 196404 (2006).

${ }^{18}$ R. Schulz, T. Korn, D. Stich, U. Wurstbauer, D. Schuh, and W. Wegshceider, Physica E (Amsterdam) 40, 2163 (2008).

${ }^{19}$ D. Grundler, Phys. Rev. Lett. 84, 6074 (2000).

${ }^{20}$ K. Morita, H. Sanada, M. Matsuzaka, C. Y. Hu, Y. Ohno, and H. Ohno, Physica E (Amsterdam) 21, 1007 (2004).

${ }^{21}$ W. Desrat, F. Giazotto, V. Pellegrini, M. Governale, F. Beltram, F. Capotondi, G. Biasiol, and L. Sorba, Phys. Rev. B 71, 153314 (2005).

${ }^{22}$ C. Heyn, S. Mendach, S. Löhr, S. Beyer, S. Schnüll, and W. Hansen, J. Cryst. Growth 251, 832 (2003).

${ }^{23}$ F. Capotondi, G. Biasiol, D. Ercolani, V. Grillo, E. Carlino, F. Romanato, and L. Sorba, Thin Solid Films 484, 400 (2005).

${ }^{24}$ D. Ercolani, G. Biasiol, E. Cancellieri, M. Rosini, C. Jacoboni, F. Carillo, S. Heun, L. Sorba, and F. Nolting, Phys. Rev. B 77, 235307 (2008).

${ }^{25}$ S. Löhr, S. Mendach, T. Vonau, Ch. Heyn, and W. Hansen, Phys. Rev. B 67, 045309 (2003).

${ }^{26}$ K. K. Choi, D. C. Tsui, and S. C. Palmateer, Phys. Rev. B 33, 8216 (1986).

${ }^{27}$ E. Zaremba, Phys. Rev. B 45, 14143 (1992).

${ }^{28}$ S. J. Papadakis, E. P. De Poortere, H. C. Manoharan, J. B. Yau, M. Shayegan, and S. A. Lyon, Phys. Rev. B 65, 245312 (2002).

${ }^{29}$ B. Habib, E. Tutuc, S. Melinte, M. Shayegan, D. Wasserman, S. A. Lyon, and R. Winkler, Phys. Rev. B 69, 113311 (2004).

${ }^{30}$ M. A. Paalanen, D. C. Tsui, and J. C. M. Hwang, Phys. Rev. Lett. 51, 2226 (1983).

${ }^{31}$ A. F. Braña, C. Diaz-Paniagua, F. Batallan, J. A. Garrido, E. Muñoz, and F. Omnes, J. Appl. Phys. 88, 932 (2000). 
${ }^{32}$ T. Ando, A. B. Fowler, and F. Stern, Rev. Mod. Phys. 54, 437 (1982).

${ }^{33}$ P. Coleridge, M. Hayne, P. Zawadzki, and A. Sachrajda, Surf. Sci. 361-362, 560 (1996).

${ }^{34}$ S. Elhamri, R. S. Newrock, D. B. Mast, M. Ahoujja, W. C. Mitchel, J. M. Redwing, M. A. Tischler, and J. S. Flynn, Phys. Rev. B 57, 1374 (1998).

${ }^{35}$ G. C. Osbourn, J. Vac. Sci. Technol. 2, 4219 (1974).

${ }^{36}$ S.-Y. Lin, H. P. Wei, D. C. Tsui, and J. F. Klem, Appl. Phys. Lett. 67, 2170 (1995).
${ }^{37}$ K. H. Hellwege, Landolt-Börnstein Numerical Data and Functional Relationships in Science and Technology: Group III: Crystal and Solid State Physics (Springer-Verlag, Berlin, 1982).

${ }^{38}$ J. C. Hensel and K. Suzuki, Phys. Rev. B 9, 4219 (1974).

${ }^{39}$ P. T. Coleridge, R. Stoner, and R. Fletcher, Phys. Rev. B 39, 1120 (1989).

${ }^{40}$ A. Gold, Phys. Rev. B 38, 10798 (1988).

${ }^{41}$ H. X. Tang, R. K. Kawakami, D. D. Awschalom, and M. L. Roukes, Phys. Rev. Lett. 90, 107201 (2003). 